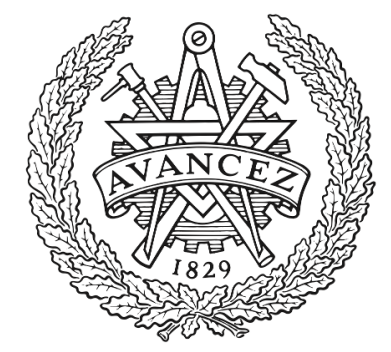

CHALMERS

UNIVERSITY OF TECHNOLOGY

\title{
CFD investigation on wheel rotation modelling
}

Downloaded from: https://research.chalmers.se, 2023-04-26 12:53 UTC

Citation for the original published paper (version of record):

Hobeika, T., Sebben, S. (2018). CFD investigation on wheel rotation modelling. Journal of Wind Engineering and Industrial Aerodynamics, 174: 241-251.

http://dx.doi.org/10.1016/j.jweia.2018.01.005

N.B. When citing this work, cite the original published paper. 


\section{Numerical Investigation on Wheel Rotation Modelling}

\section{Teddy Hobeika and Simone Sebben}

Chalmers University of Technology

\section{Highlights}

\section{Abstract} vehicle.
- Moving Reference Frame - grooves (MRFg) approach for modelling tyre rotation is presented.

- It is validated on a freely rotating isolated wheel against the sliding mesh approach.

- The prediction of tyre pattern modifications using MRFg shows good agreement with experimental measurements on a full scale

It is well established in the automotive community that wheels are a major contributor to the aerodynamic drag of passenger vehicles. The flow around rotating wheels is very complex due to the many separation regions created by very small tire features and by the contact area of the tire with the ground. Correct modelling of wheel rotation requires accuracy in the representation of the tire geometry and proper boundary conditions to simulate the rotation. This paper proposes a boundary condition that simulates tire rotation which is simple to implement and does not suffer from the limitations of a sliding mesh approach at the region where the tire meets the ground. The method is first evaluated on a single wheel that is free standing and the results are compared to a full sliding mesh computation, which is considered to be the best possible numerical solution. The technique is then implemented on a complete vehicle model simulation and the results are correlated against wind tunnel force measurements. The good agreement obtained in these tests cases show that the proposed boundary condition is a promising solution to a more accurate numerical simulation of rotating wheels.

\section{Introduction}

Governmental regulations and increased consumer awareness of the effects of global warming has led the automotive industry to maximize its efforts to improve the energy efficiency of its fleet. In this development, the aerodynamic drag is a fundamental parameter to minimize since it has a direct link to the fuel consumption. Although much effort is put into improving the aerodynamic characteristics of the car exterior, it is well known that the wheels of the vehicle contribute to approximately $25 \%$ of the overall drag. For this reason, over the past years, wheel aerodynamics has been receiving special attention within the academia and the industrial research. The flow around the wheel area is highly unsteady and complex with many regions prone to separation. To understand this flow and its influence on the total forces of the vehicle, aerodynamicists have tried in the past to isolate the effects of wheels by separating them into two components: the rims and the tires.

Several numerical and experimental investigations have been dedicated to the survey of different rim designs and its effect and interaction with the surrounding flow such as [1-4]. Most of such studies used simplified tyres without a pattern, slicks, or used the same tyre for all rim configurations. However, studies doing different combinations of tyre and rim designs have found evidence of an interaction between the 
two making it difficult to study their effects independently from each other [5-7].

Many studies have also been dedicated to the understanding of the significance of tire geometry on the aerodynamic drag of vehicles.

Numerically, investigations of rotating tires are particularly challenging since proper computation of the rotational condition is difficult due to the many complex and small tire features, the area at contact with the ground, and the fact that the tire suffers deformation as a result of weight loads and centrifugal forces. Some papers looked at isolated wheels with various contact patch sizes $[8,9]$, or profile curvatures and camber angles $[10,11]$, while others looked at the tyre effect in combination with the vehicle flow field $[6,12]$.

All of the mentioned numerical studies struggle to achieve correct simulation of the rotational condition of the tyre pattern and instead utilize a slick tyre, or simply overlook the pattern modelling. A correct simulation is one in which the mesh of the rotating parts slide, or move, accordingly to the speed of rotation. Although easy to implement for the rims, the sliding mesh condition is not feasible to implement for the tires due to the area of contact with the ground. At the ground, the tire is deformed due to vehicle load and loses its complete circular form. The side wall bulges out, the tyre merges with the ground forming a contact patch, and the pattern is squeezed and distorted. A view of the problem can be seen in Figure 1.

Different alternatives have been investigated to resolve this issue, like for example avoiding the moving mesh problem by removing the tyre pattern and replacing it with a numerical surface roughness applied to the tyre surface[13]. Other approaches looked into keeping the tyre with its full details and circular form whilst implementing an Immersed Boundary approach to simulate the rotation as it goes through the ground[14]. This allows for the movement of the mesh, however it results in a significantly over sized contact patch and does not capture the surface friction at the immersed boundary.

The focus of this paper is on the implementation and validation of a

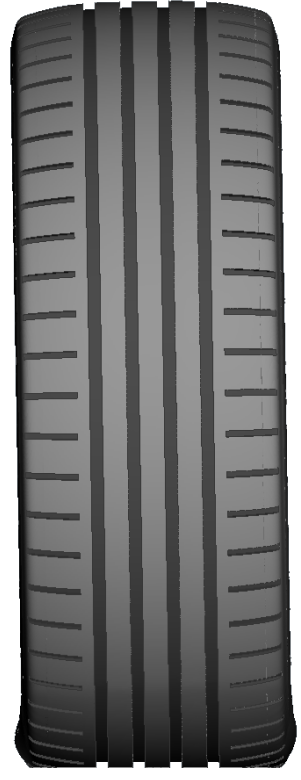

(a) Front view

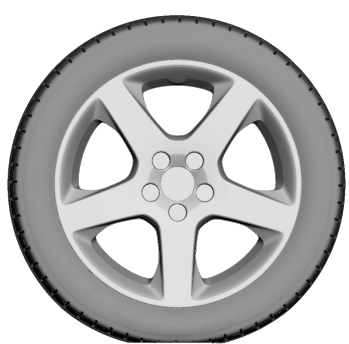

(b) Side view

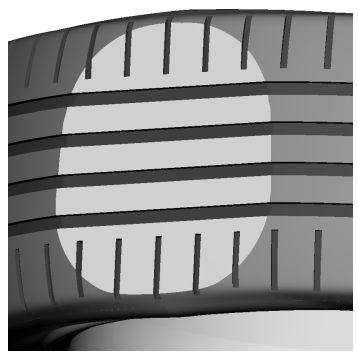

(c) Contact patch view
Figure 1: Tyre deformation under load on a passenger car.

new boundary approach to simulate the rotational behavior of the tire which overcomes the limitations of the sliding mesh approach. An early version of the this approach has been previously looked into by Hobeika et al. [15] and showed promising results, however it lacked thorough analysis and validation. The current improved version is presented and validated on a single, free standing rotating wheel where a fully sliding mesh approach is possible for comparison. Later the method is tested against the traditional rotating wall approach in a complete vehicle simulation and its predictive abilities for various wheel configurations are compared to experimental results.

\section{Methodology}

This section mainly describes the numerical approach used to simulate the rotation of tyres. Most Computational Fluid Dynamics (CFD) codes offer different numerical approaches to describe models with rotational parts: Rotating Wall (RW), Moving Reference Frame (MRF) and Sliding Mesh (SM). These approaches are briefly described here in order to introduce the MRFg (Moving Reference Frame - grooves) approach. 
The MRFg validation is performed in two steps. First, the method is validated against sliding mesh simulations on an isolated wheel and then the aerodynamic effects are analyzed on a fully detailed vehicle and compared to full-scale wind tunnel data.

The geometry of the tires investigated, as well as that of the complete vehicle are reviewed in this section. This is followed by the numerical and experimental setup.

\subsection{Rotation Modelling}

The Rotating Wall boundary condition is one of the most common approaches for modelling rotating parts. This is implemented through the introduction of a velocity term at the wall which is tangential to the cell surface. Due to conservation of mass, the velocity cannot have a component normal to the cell surface as this would be physically interpreted as in/outflow through a solid wall. Given the geometrical complexity of rims and tyres, many surfaces would not be modeled correctly with RW as their movement is in a direction normal to the surface. This can be seen in Figure 2a where the faces in the tyre lateral grooves and inside the rim spokes show a lower velocity (in yellow) than the faces aligned tangentially to the velocity vector. Figure $2 b$ shows the correct velocity distribution on the wheel for comparison.

The Moving Reference Frame approach is able to overcome this problem by setting the fluid as part of a local rotating reference frame with respect to the global reference. This introduces centrifugal accelerations and Coriolis effects into the fluid. The approach is widely used as an approximation to rotating parts such as in the case of fans and wind turbines $[16,17]$. However, the size of the MRF region has a significant effect on the overall results, as it determines the amount of rotation introduced into the flow. In certain cases, a strong pressure gradient is also introduced as presented by Hobeika et al. [15]. Additionally, as the mesh is fixed in the MRF region, the position of the rotating parts will have a clear local imprint on the flow which could give misleading

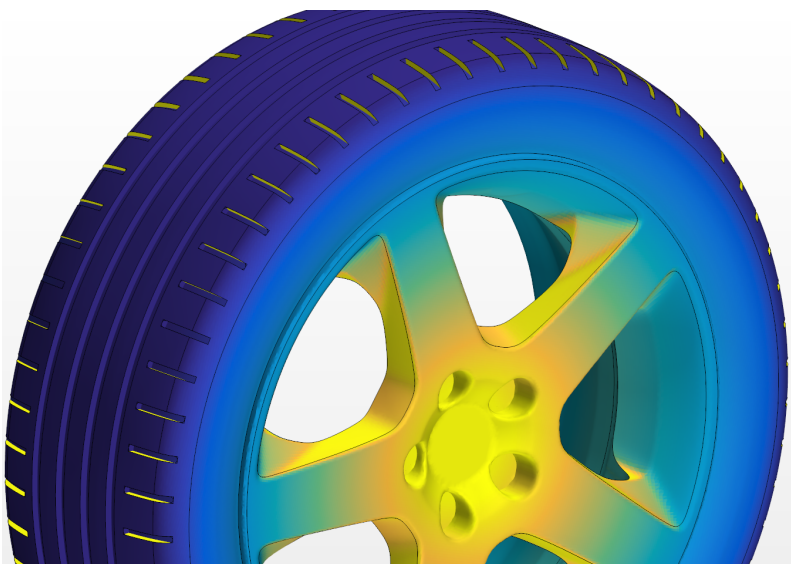

(a) Rotating wall boundary condition

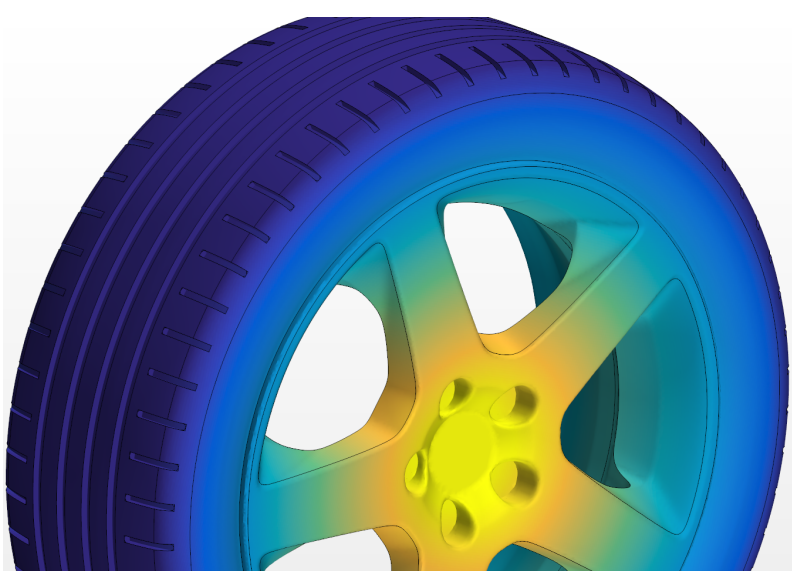

(b) Correct velocity distribution

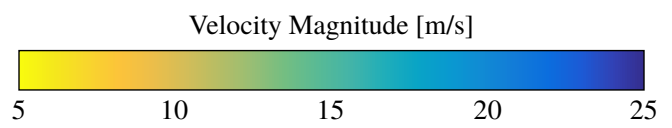

Figure 2: Velocity distribution on a wheel obtained through Rotating Wall condition vs. the correct distribution.

results.

The most realistic modelling method to work around these challenges is to literally move the mesh. Therefore an unsteady simulation setup is required with the mesh physically rotating every time step. This is commonly known as Sliding Mesh and is implemented as a rigid body motion, hence easily applied to rims. However in the case of tyres, and given the deformation they experience while rotating as well as their contact with the ground as shown in Figure 1, SM is quite challenging. Furthermore, moving the mesh every time step and interfacing it to the neighboring fixed cells, comes at a high computational expense and 
leads to significant increase in run time.

The MRFg method works around moving the mesh and does not affect the total simulation run time. The method combines rotating wall and moving reference frame approaches by utilizing advantages from both RW is utilized on the external tyre area, where the rotational velocity correctly translates into a tangential component on the surface, while MRF is applied in the tyre lateral grooves. The mesh is still fixed and hence MRFg still it is not able to take into account all various tyre positions. However unlike the rim spokes, the tyre latyeral grooves are small in size and very repetitive, thus the local flow differences are not expected to change the overall results. This is further elaborated on in Section 5 .

\subsection{Isolated Wheel Setup}

The isolated wheel setup consists of an isolated wheel with a closed rim design which is rotating in free stream away from any surface influence. The aim of this set up is to generate a flow field around the wheel driven primarily by the wheel rotation. The wheel rotational speed is set to $90 \mathrm{rad} / \mathrm{s}$ which is close to the speeds the wheel experiences when mounted on a passenger car driving at $100 \mathrm{~km} / \mathrm{h}$. The free stream velocity is set to a value close to zero $(1 \mathrm{~km} / \mathrm{h})$ so as not to have a significant influence on the flow field around the wheel but merely to flush the domain towards the outlet.

In this setup, the wheel is rotationally symmetric and hence can be modelled using sliding mesh (SM). As SM simulates the true wheel rotation by physically sliding the mesh each time step, it is considered an accurate rotation modelling method for CFD applications. The results from SM are used as the reference for rotation modelling.

For the purpose of validation, the ventilation moment, the moment resisting the wheel's rotation, is used to quantify the impact of the wheel on the flow around it. The ventilation moment is this setup with almost no air flow is the equivalent of the "zero ventilation" presented

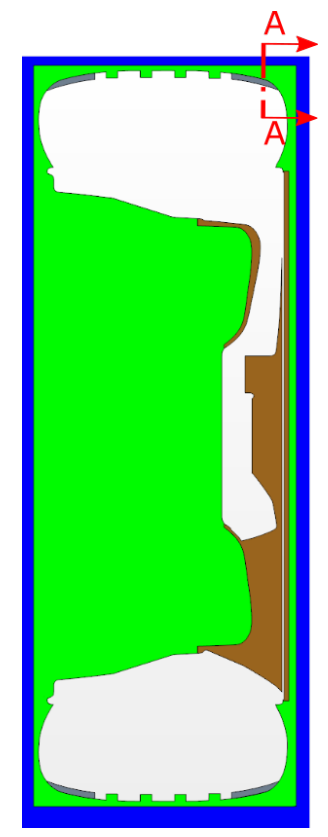

(a) Front view (b) A-A cross section

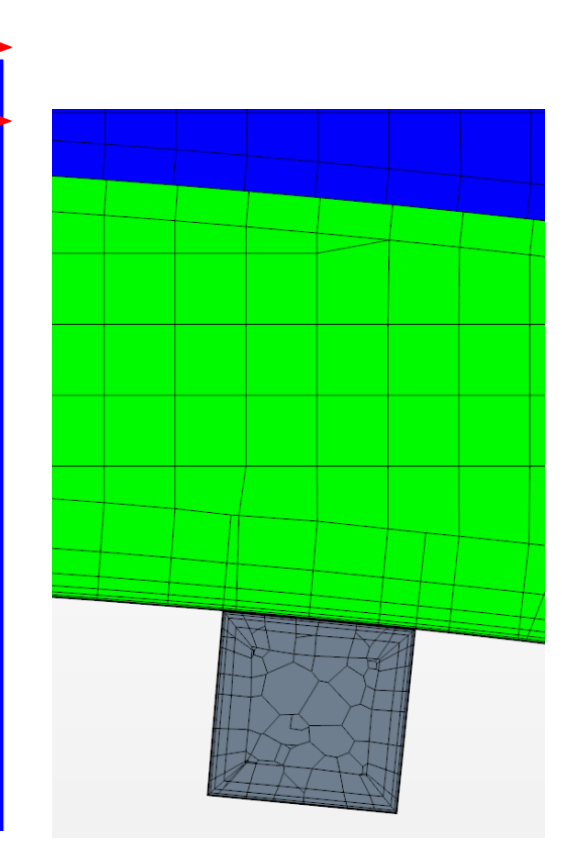

Figure 3: Region distribution and mesh representation: Region 0 in blue, Region 1 in green, Region 2 in brown, and Region 3 in gray.

by Wickern et al. [5].

The simulations investigated two tyre designs of same size and profile: a slick (S) and a fully detailed tyre (D).One mesh was generated for each tyre in a way that all rotation modelling methods could be performed, thus avoiding any mesh reproducibility effects. The mesh settings used on the wheels resulted in a maximum surface size on $2 \mathrm{~mm}$ and a first cell height of $0.01 \mathrm{~mm}$ with a slow growth into the volume. Figure 3 shows how the volume mesh is split into three separate regions, Regions 0,1 , 2, and 3. Region 0 contains the magority of the computational domain but no wheel parts, Region 1 includes the complete wheel geometry, Region 2 isolates the rim spokes from the wheel geometry, and finally Region 3 contains the tyre lateral grooves. Naturally, Region 3 only exists when the lateral grooves are present, ie. for tyre D. presented below: 
- S2: Sliding mesh on Region 2 and rotating wall on all surfaces in Region 1.

- S3: Sliding mesh on both Region 1 and 2.

- S4: Sliding mesh on Region 1 and rotating wall on all surfaces in Region 2.

The different modelling approaches investigated for the detailed tyre are presented below:

- D1: Rotating wall on all wheel surfaces in both Regions 1,2 and 3.

- D2: Sliding mesh on Region 2 and rotating wall on all surfaces in Region 1 and 3.

- D3: Sliding mesh on Region 1, 2, and 3.

- D4: Rotating wall on all surfaces in both Region 1 and 2, but with MRF on Region 3.

- D5: Sliding mesh on Region 2, rotating wall on all surfaces in Region 1 and MRF on Region 3.

All the modelling approaches mentioned above are summarized in Table 1.

\section{$4.3 \quad$ Full Vehicle Setup}

The objective behind the vehicle setup is to investigate the force predictions on a vehicle when MRFg is utilized and compare to measured experimental data. The traditional RW approach for tyre rotation modelling is also investigated in order to quantify improvements in prediction capability. For this purpose, in both wind tunnel tests and numerical experiments, three tyre patterns were tested on a sedan vehicle: a slick tyre (S), a lateral grooved tyre $(\mathrm{G})$ and a detailed tyre (D). Figure 4 shows a geometry representation of the tyres.

All three tyre sets were initially slick tyres of the same dimensions, which

\begin{tabular}{llll}
\hline Approach & R1 & R2 & R3 \\
\hline \hline S1 & $\mathrm{RW}_{\text {(surfaces) }}$ & $\mathrm{RW}$ & $\mathrm{N} / \mathrm{A}$ \\
S2 & $\mathrm{RW}$ & $\mathrm{SM}_{\text {(region) }}$ & $\mathrm{N} / \mathrm{A}$ \\
S3 (REF) & $\mathrm{SM}$ & $\mathrm{SM}$ & $\mathrm{N} / \mathrm{A}$ \\
S4 & $\mathrm{SM}$ & $\mathrm{RW}$ & $\mathrm{N} / \mathrm{A}$ \\
\hline D1 & $\mathrm{RW}$ & $\mathrm{RW}$ & $\mathrm{RW}$ \\
D2 & $\mathrm{RW}$ & $\mathrm{SM}$ & $\mathrm{RW}$ \\
D3 (REF) & $\mathrm{SM}$ & $\mathrm{SM}$ & $\mathrm{SM}$ \\
D4 & $\mathrm{RW}$ & $\mathrm{RW}$ & $\mathrm{MRF}$ \\
D5 (MRFG) & $\mathrm{RW}$ & $\mathrm{SM}$ & $\mathrm{MRF}$ \\
\hline
\end{tabular}

Table 1: A summary of the various rotation modelling approaches investigated on the isolated wheel

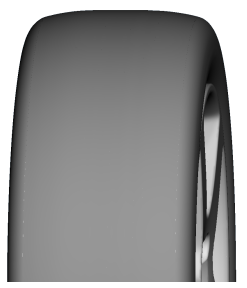

(a) Slick tyre (S)

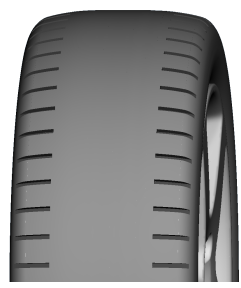

(b) Grooved tyre (G)

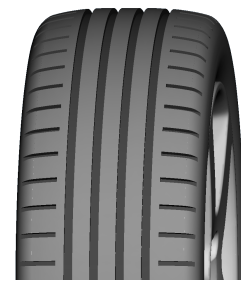

(c) Detailed tyre (D)
Figure 4: Geometry representations of the tyres.

had grooves cut into two of the tyre sets according to specified dimensions with good accuracy and reproducibility. The groove dimensions are chosen in a way to resemble realistic tyres while keeping the geometry simple enough to produce a good quality mesh. By cutting the tyre patterns on demand into the same slick tyres, it has been ensured that the tyre outer profile, sidewall curvature, deformation under load, and position with respect to the rim are practically identical. The tyres were originally designed for racing and thus are extremely stiff and show negligible deformation due to rotation especially since the test vehicle is modified to have a rigid suspension, thus allowing good control of the tyre's position inside the wheelhouse and the wheel center height above from the ground. In order to replicate the tyre geometry while mounted on a rim and deformed under the load of the car, 3D scans and various 


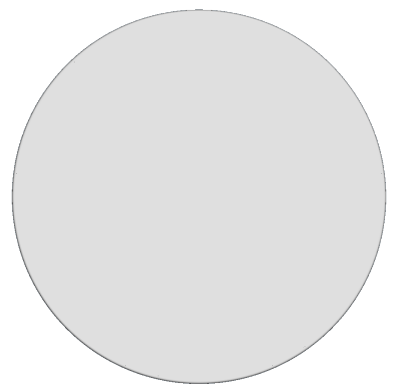

(a) Closed rim (CR)

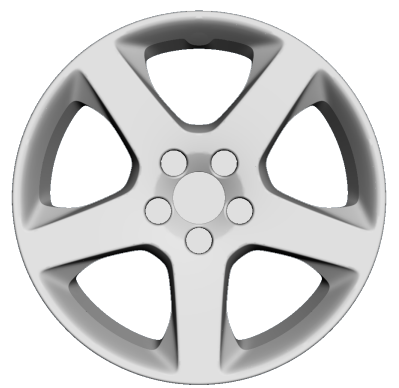

(b) Open rim (OR)

Figure 5: Geometry representations of the rims.

\subsection{Numerical Setup}

The simulations are performed in StarCCM+ and using a hybrid RANS-DES solver. The RANS part is applied at the boundaries with the k-omega SST model while the detached unsteady separated flow is solved using the DES. The formulation used is the Improved Delayed Detached Eddy Simulation (IDDES) presented in [18], with 2nd order temporal discretization and 2nd order upwind spatial discretization. The simulations are averaged over the last $2 \mathrm{~s}$ of a $5 \mathrm{~s}$ physical run time with a time step of $2 \times 10^{-4} \mathrm{~s}$, which results in a convective Courant number below 5 in most cells in the domain. An investigation of increasing averaging time up to $4 \mathrm{~s}$ of a $7 \mathrm{~s}$ physical run time showed negligible changes to the mean flow field and to the forces acting on the vehicle. Similarly, an investigation into reducing the time step to $1 \times 10^{-4} \mathrm{~s}$ and $5 \times 10^{-5} \mathrm{~s}$ resulted in minor changes to the flow field with slight changes in overall drag and its distribution over the vehicle. The drag in measured in terms of a dimensionless coefficient $\left(\mathrm{C}_{\mathrm{d}}\right)$ which varied by significantly less that $1 \%\left(0.002 \mathrm{C}_{\mathrm{d}}\right)$ for all setup investigations including a mesh dependency study.

The mesh sizes for the isolated wheel and full vehicle setup averaged about 10 and 130 million cells, respectively. Prism layers with a first cell height of $0.01 \mathrm{~mm}$ were built on all wheel surfaces and exterior vehicle surfaces which were in direct exposure with the main flow thus achieving a y+ value well below one.

\subsection{Experimental Setup}

All experimental measurements were conducted in the full scale Volvo Cars Aerodynamic Wind Tunnel (PVT) and at a speed of $100 \mathrm{kph}$. The tunnel is a closed loop type with a slotted wall test section and a cross sectional area of $27 \mathrm{~m}^{2}$. To simulate road flow conditions around the vehicle, a boundary layer control system (BLCS) is available that includes a five-belt moving ground system. The tunnel has an 
uncertainty in $\mathrm{C}_{\mathrm{d}}$ measurements of 0.001 within the same test and it is accredited according to the European Accreditation procedure EA 4/02 [19].

\section{Results and Discussion}

The results from the isolated wheel and full vehicle studies are presented and discussed in this section.

\subsection{Isolated Wheel}

As previously discussed, the ventilation moment and its distribution over the various parts is utilized to quantify the wheel's rotational effect on the flow field. It can be split on the various wheel parts to isolate the effects of different methods and get a more detailed understanding of its development. It is thus split on the rim, tyre, and grooves, furthermore the contributions from pressure and viscous resistances are also identified.

Figure 6 summarizes the part specific ventilation moments for the different modelling methods for the slick tyre. S3 is the fully SM method and hence the goal of any other rotation modelling method is to replicate its results. It is clear that $\mathrm{S} 2$ is the only modelling method able to reproduce the fully Sliding Mesh results, both in total wheel moment and the part contributions. It is worth noting that the tyre contribution to ventilation moments is well predicted in all methods. The rim's contribution on the other hand, can only be correctly predicted when the rim is modeled using SM, as is the case in S2 and S3.

In Figure 7, the velocity field inside the rim and around the tyre are presented along with convoluted streamlines. The velocity close to the tyre is very similar for all four modelling methods which correlates well with the ventilation moments. This is expected for the slick tyre as the tyre surface velocity is mostly tangential to the tyre surface and this can be reproduced using RW. The velocities around the rim for S2 and S3

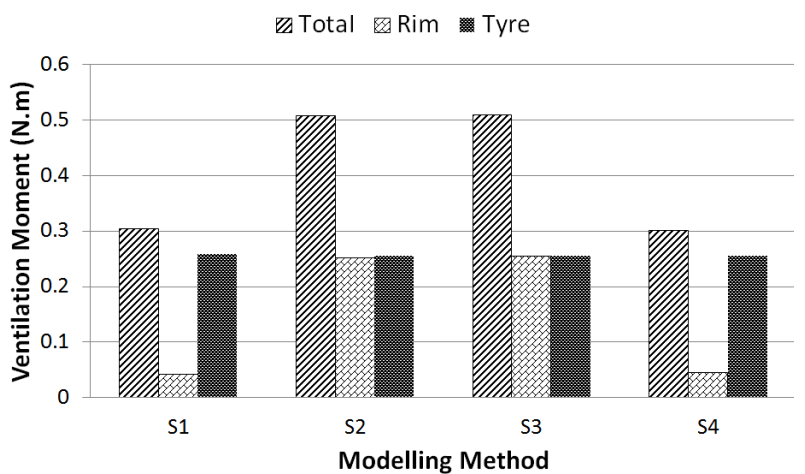

Figure 6: Results of ventilation moments by parts for a wheel with slick tyre. of a slick tyre while SM is necessary for modeling the rim rotation.
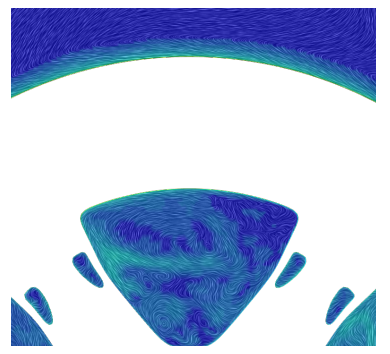

(a) S1: RW rim and tyre
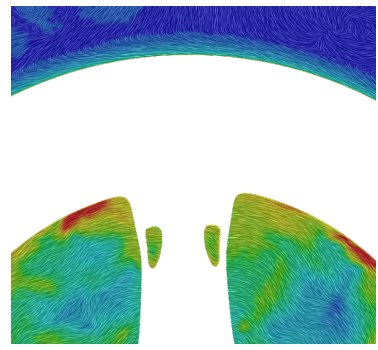

(c) S3: SM rim and tyre

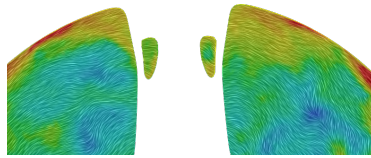

(b) S2: SM rim, RW tyre
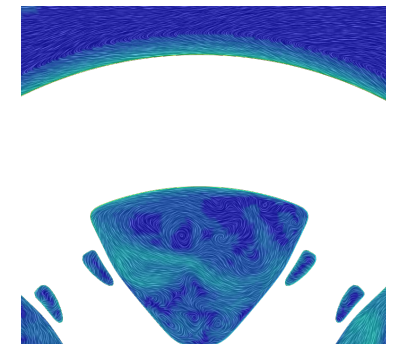

(d) S4: RW rim, SM tyre

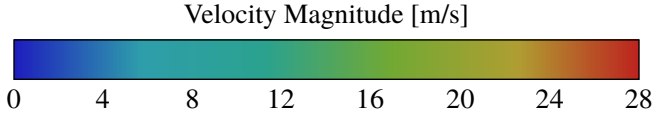

Figure 7: Velocity field in a plane passing through the rim spokes of a closed rim with a slick tyre. 

when MRFg is applied on the lateral grooves as in D4 and D5.

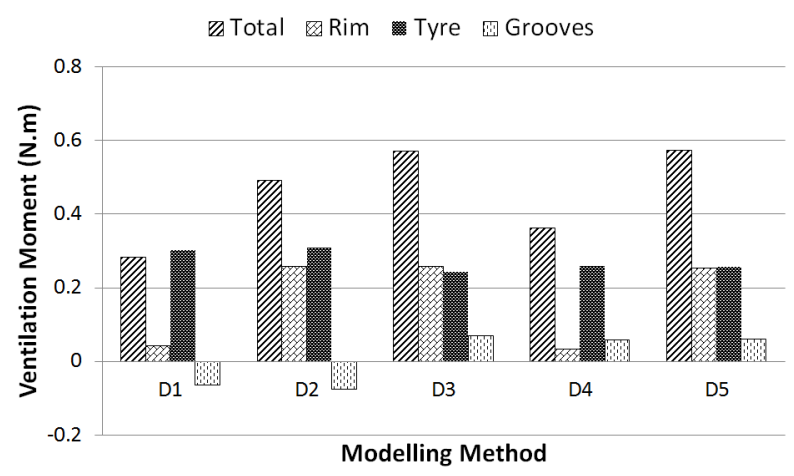

Figure 8: Results of ventilation moments by parts for a wheel with detailed tyre.

Similarly to the slick tyre, the ventilation moments results for the detailed tyre are presented in Figure 8, with the contribution of the lateral grooves separately presented. D3 is the fully SM method and therefore considered to be the correct solution. It can be seen from the figure that D5 (MRFg) is the only method that is able to reproduce the results, both in total wheel moment and in part contributions. D2 comes close in total moment prediction, however it falls short of predicting the contribution attributed to the lateral grooves. Furthermore, the lateral groove contribution is incorrectly predicted as a negative ventilation moment when RW is used, in D1 and D2. This was expected as the lateral grooves modelling was previously identified as a weaknesses of Rotating Wall. The SM tyre results, D3, are only correctly reproduced

The conclusions from the slick tyre simulations still hold regarding the rim modelling and the remaining of the tyre surface (excluding the lateral grooves). The Sliding Mesh effect on the rims clearly stand out in D2, D3 and D5, showing again its necessity for accurate simulations and highlighting them as three key simulations for closer flow field investigations. The difference in the velocity fields locally around one of the grooves can be seen in Figure 9. Methods D2, D3, and D5 are presented to highlight the modelling effects of RW, SM , and MRFg, respectively. The low velocity displayed inside the groove for D1, Figure $9 \mathrm{a}$ is concerning as it is in such proximity of a rotating surface. Furthermore, the high velocity on the tyre surface to the left side of the grooves dies out as the flow passes over it and results in a low velocity

on the tyre surface to the right side of the groove. This is not seen from D3 and D5 where the fluid inside the groove shows a high velocity of similar magnitude to the tyre's tangential velocity. Figure $9 b$ and Figure $9 \mathrm{c}$ also show how the high velocity at the tyre surface left of the groove is preserved over to the right side of the groove. This shows that MRFg is able to closely predict the flow field in the vicinity of the lateral grooves and is able to reproduce the ventilation moment on the wheel as SM.

MRFg differs from SM as the mesh geometry is fixed, hence although the global effect could be reproduced to a good extent, some local variances at the tyre should be present. One such difference can be seen in Figure 10 when comparing the different contributions of pressure and shear to the ventilation moment of the complete wheel. Only a handful of approaches are presented in Figure 10 as these have shown to be the most relevant ones for the validation. For the slick tyre, the fully sliding mesh, $\mathrm{S} 3$, is presented along with the combination of sliding mesh on the rim spokes and rotating wall on the rest of the wheel surfaces, S2. The good match in contributions further supports the conclusion that $\mathrm{RW}$ on a slick tyre is sufficient to reproduce SM results.

For the detailed tyre, the three configurations with Sliding Mesh on the rim spokes are presented, hence they mainly differ in how the tyre and lateral grooves are modelled. D2, with rotating wall around the tyre and lateral grooves, shows a poor prediction of teh distribution compared to D5, the fully sliding mesh, which was expected given the poor flow prediction around the lateral grooves as shown previously in Figure 9. D5, with MRFg, shows close but not spot on, results compared to D3, with the fully SM, even though the part specific ventilation moments, presented in Figure 8, and local flow field pictures, presented in Figure 9, matched quite well. In order to understand where such deviations come from, the pressure distribution on the tyre surface is investigated. Figure 11 shows how the pressure distribution in the lateral grooves is significantly different between $\mathrm{D} 2, \mathrm{RW}$ approach, and $\mathrm{D} 3, \mathrm{SM}$ approach, proving again the inaccurate modelling of the rotating wall 


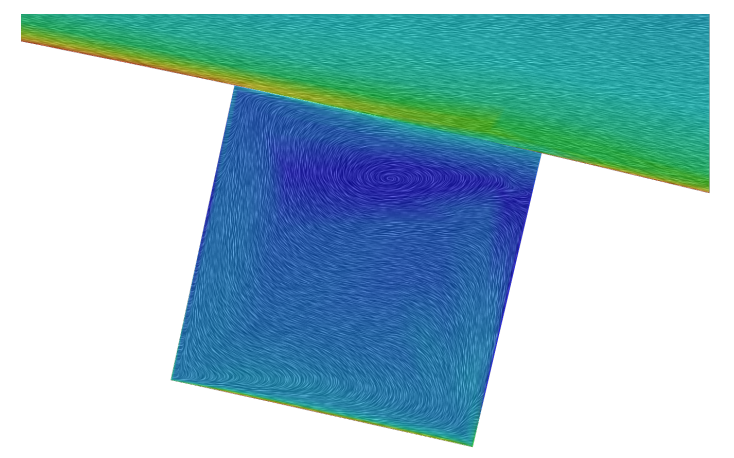

(a) D2 - RW the tyre and grooves

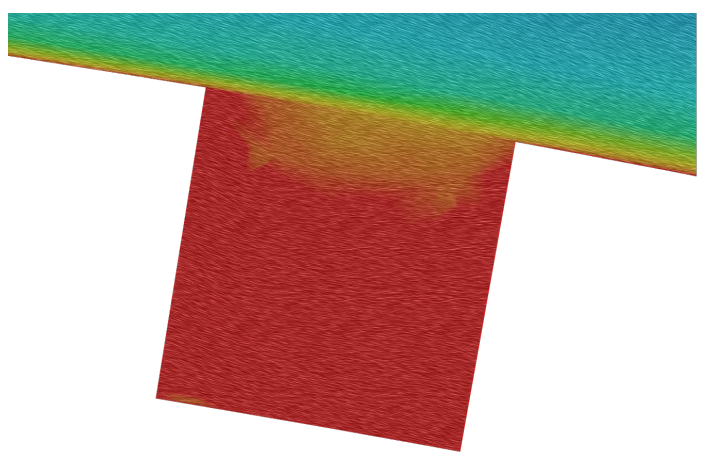

(b) D3 - SM both tyre and grooves

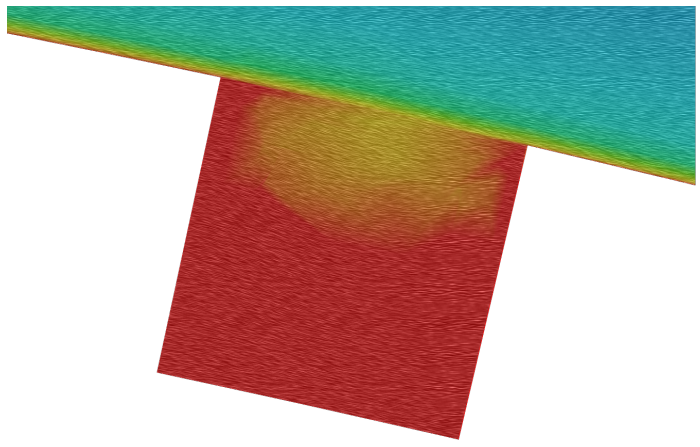

(c) D5 - MRFg: RW tyre and MRF grooves

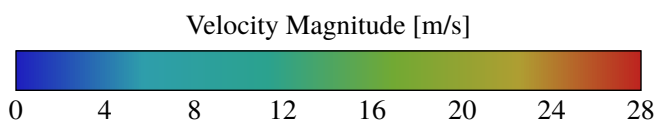

Figure 9: Velocity field in a plane cutting through one of the lateral grooves. The clockwise tyre rotation drives the flow left to right along the tyre surface.

approach. The pressure inside the grooves is a high positive pressure in D2,Figure 11a, while it should be a low negative pressure as shown in D3,Figure 11b. The MRFg approach, Figure 11c, is able to give a similar pressure within the groove to D3 however the pressure prediction on the surface of the tyre is not exactly the same. This is one of the consequences of having a fixed mesh, where the interaction between the

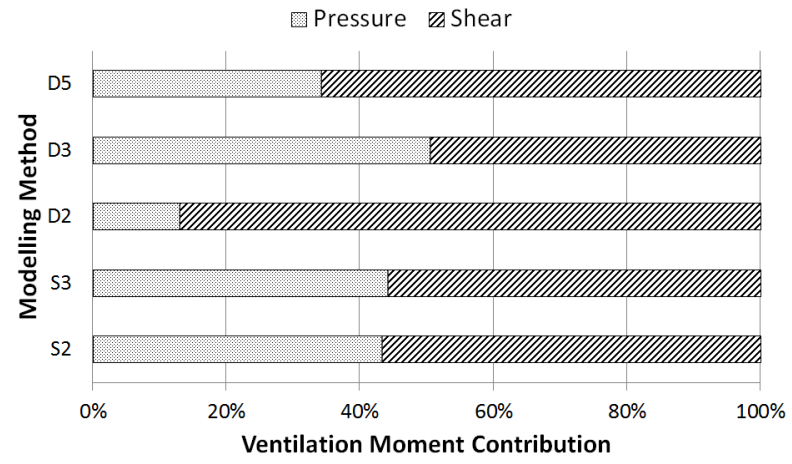

Figure 10: Results of the percentage contributions of pressure and shear forces to the overall wheel ventilation moment.

groove and the surfaces around it cannot be perfectly replicated even though the overall effect on the flow field is well predicted.

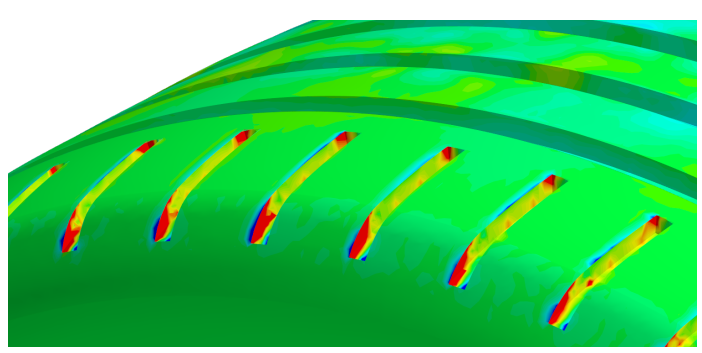

(a) D2 - RW the tyre and grooves

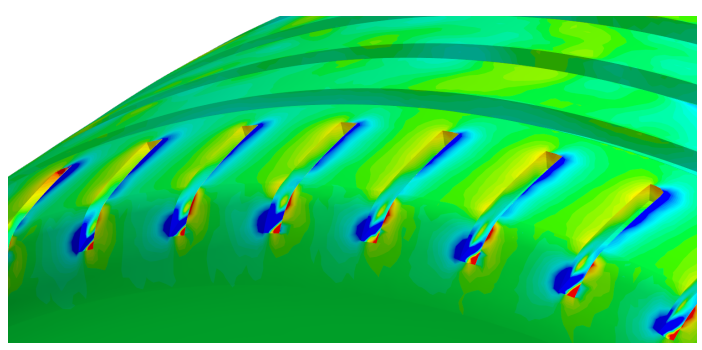

(b) D3 - SM both tyre and grooves

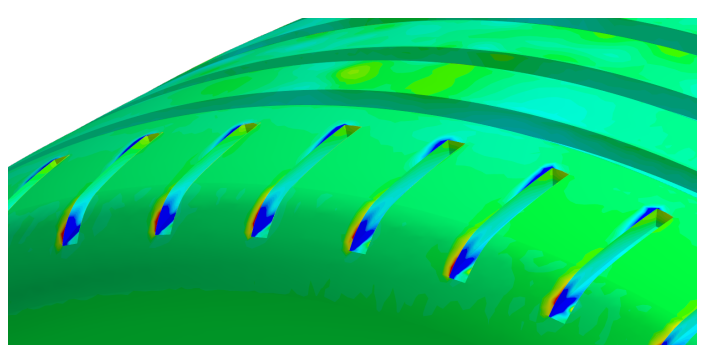

(c) D5 - MRFg: RW tyre and MRF grooves

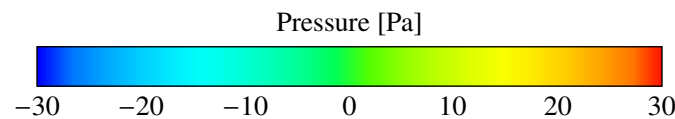

Figure 11: Pressure distribution on the tyre surface and in the lateral grooves. 
$\Delta \Delta C_{d X}=\left(C_{d X}-C_{d \text { Slick }}\right)_{\text {simualtion }}-\left(C_{d X}-C_{d \text { Slick }}\right)_{\text {experiment }}$

The bars represent the ability of the simulation method to reproduce the

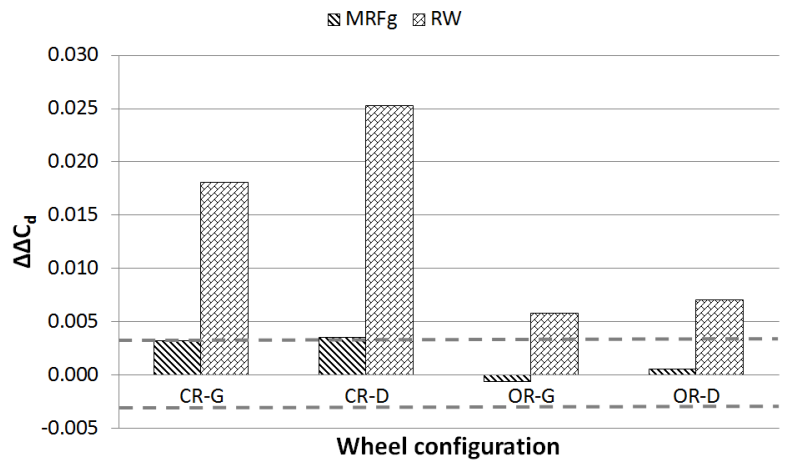

Figure 12: Results Moments for detailed tyre.

\subsection{Full Vehicle}

The results presented in this section show the differences in drag forces and flow structures resulting from modelling the tyre rotation with Rotating Wall and MRFg. This is quantified by comparing the changes in overall vehicle drag when changing from a slick tyre to the lateral grooved and detailed tyres. The change predicted from the simulations is subtracted by the respective change predicted from experiments. This is expressed in Equation 1, and when calculated for all four tyre and rim combinations simulated with RW and MRFg, eight values are obtained and presented in Figure 12.

experimental trends when adding details on a slick tyre for a closed and an open rim. The two dotted lines highlight the uncertainty $(\sigma=0.003)$ margins of this comparison based on the experimental measurement uncertainty of $0.001 \mathrm{C}_{\mathrm{d}}$ and the simulation setup variability of 0.002 $\mathrm{C}_{\mathrm{d}}$, estimated from the investigations in Section 4.4. From the results, it can be seen that the modelling of lateral grooves using RW leads to extremely misleading results more than $8 \sigma \mathrm{s}$ off for a closed rim and $2 \sigma \mathrm{s}$ for an open rim. By applying MRFg this incorrect prediction could be reduced to almost one $\sigma$ for a closed rim and well within uncertainty margins for the open rim case.

Figure 13 shows the isosurface of Q-criterion at 5000/s ${ }^{2}$ at the front left wheel colored by vorticity magnitude. The flow structures look very similar for the slick tyre, Figure 13a, and grooved tyre with MRFG implementation, Figure 13b. As the oncoming flow goes around the slick tyre, small vortices are generated when it reaches the closed rim, while for the grooved tyre with MRFg, similar vortices are generated further upstream around the lateral grooves. These vortices are of similar size and in both cases they merge into the larger vortex structure created from the contact patch. When RW is implemented on the grooved tyre, a much stronger separation is created with large structures that merge together to form a large sheet covering a large part of the wheel, as shown in Figure 13c. This results in an increase in drag which is significantly larger than the experimental results, as shown in Figure 12.

It is also worth noting that with RW the large vortices have an upwash direction towards the wheel house and are not entrained towards the ground by the wheel rotation as is the case for MRFG. From the isolated wheel it was clear that the lateral grooves lack the correct modelling, and even though small in size, they introduce big vortices into the flow resulting in significant drag over prediction.

\section{Conclusion}

A study of the effects of different tyre rotation modelling methods has been presented in this paper. The majority of the study is performed on an isolated wheel and the different modelling methods are validated to the fully sliding mesh approach. Furthermore, a comparison of the effects of tyre modelling on the drag prediction of various wheel configurations on a full-scale passenger car is also presented. The following key points can be concluded from this work:

- Rotating Wall can predict similar results to SM when the rotational boundary condition imposed on the surface cells is in fact tangential to the surface, which is the case for a slick tyre.

- In the case of detailed tyres, Rotating Wall predicts incorrect results due to the normal surface alignment to the tangential 


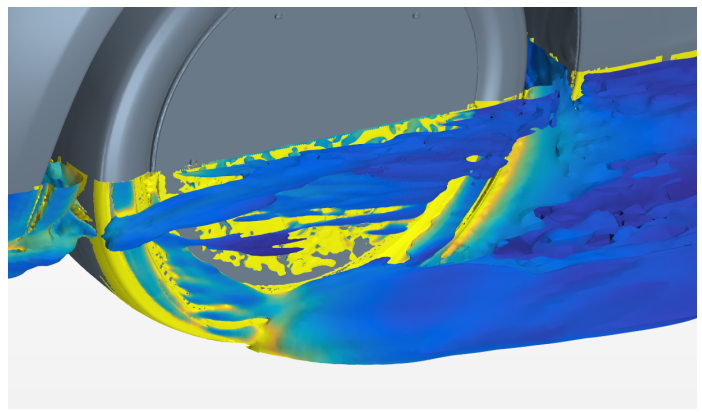

(a) CR-S: Closed rim slick tyre with RW

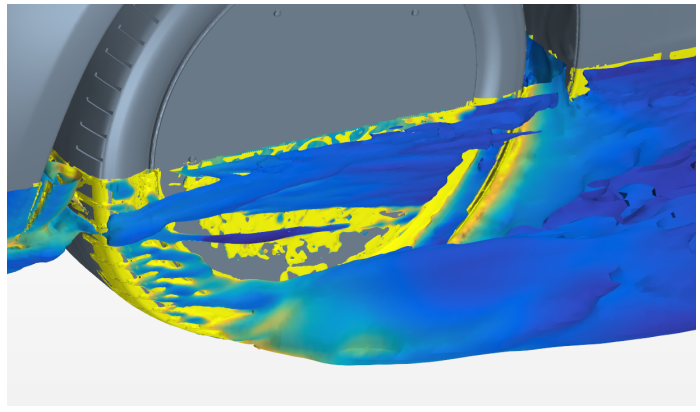

(b) CR-G: Closed rim grooved tyre with MRFG

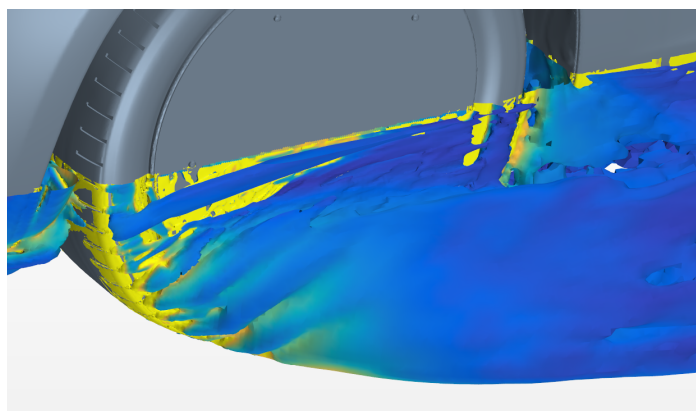

(c) CR-G: Closed rim grooved tyre with RW

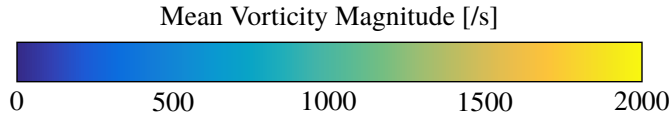

Figure 13: Isosurface of Q-criterion at 5000/s $\mathrm{s}^{2}$ colored by vorticity magnitude for the closed rim configurations.

velocity component.

- The investigated MRFg method, is able to reproduce Sliding Mesh results on an isolated wheel setup and predict tyre pattern modifications with good agreement to experiments.

- The MRFg method, also does not introduce computational costs and could be implemented for various complex geometries in steady and unsteady simulations.

\section{References}

[1] Wäschle, A., “The Influence of Rotating Wheels on Vehicle Aerodynamics - Numerical and Experimental Investigations", SAE World Congress \& Exhibition, (SAE International, Apr. 2007), DOI: $10.4271 / 2007-01-0107$.

[2] Landström, C., Löfdahl, L., Josefsson, L., and Walker, T., “An experimental investigation of wheel design parameters with respect to aerodynamic drag", 8th FKFS Conference-Progress in Vehicle Aerodynamics and Thermal Management, (Stuttgart, 2011).

[3] Koitrand, S., Gaylard, A., and Fiet, G. O., “An Investigation of Wheel Aerodynamic Effects for a Saloon Car", Progress in Vehicle Aerodynamics and Thermal Management: Proceedings of the 10th FKFS-Conference, Reihe Technik, (2015), ISBN: 9783816933229

[4] Haag, L., Blacha, T., and Indinger, T., "Experimental Investigation on the Aerodynamics of Isolated Rotating Wheels and Evaluation of Wheel Rotation Models Using Unsteady CFD”, International Journal of Automotive Engineering 8(1): 7-14, 2017, DOI: 10.20485/jsaeijae.8.1_7.

[5] Wickern, G., Zwicker, K., and Pfadenhauer, M., "Rotating Wheels - Their Impact on Wind Tunnel Test Techniques and on Vehicle Drag Results", SAE International Congress and Exposition, (SAE International, Feb. 1997), Dor: 10.4271/970133.

[6] Hobeika, T., Sebben, S., and Landstrom, C., "Investigation of the Influence of Tyre Geometry on the Aerodynamics of Passenger Cars”, SAE Int. J. Passeng. Cars - Mech. Syst. 6: 316-325, 2013, ISSN: 1946-4002, DOI: 10.4271/2013-01-0955.

[7] Landstrom, C., Josefsson, L., Walker, T., and Lofdahl, L., “Aerodynamic Effects of Different Tire Models on a Sedan Type Passenger Car", SAE Int. J. Passeng. Cars - Mech. Syst. 5: 136-151, Apr. 2012, DoI: 10.4271/2012-01-0169. 
[8] Diasinos, S., Barber, T. J., and Doig, G., "The effects of simplifications on isolated wheel aerodynamics", 50 Journal of Wind Engineering and Industrial 505 Aerodynamics 146: $90-101, \quad 2015, \quad$ ISSN: 0167-6105, ${ }_{506}$ DOI: http://dx.doi.org/10.1016/j.jweia.2015.08.004

[9] Mlinaric, P. and Sebben, S., "Investigation of the Influence of Tyre Deflection and Tyre Contact Patch on CFD Predictions of Aerodynamic Forces on a Passenger Car", MIRA International Conference on Vehicle Aerodynamics, (2008).

[10] Sprot, A. J., Sims-Williams, D. B., and Dominy, R. G., "The Aerodynamic Characteristics of a Fully Deformable Formula One Wind Tunnel Tyre", SAE International Journal of Passenger Cars - Mechanical Systems 5(2): 1026-1041, Apr. 2012, ISsN: 1946-4002, DOI: 10.4271/2012-01-1166.

[11] Schnepf, B., Tesch, G., and Indinger, T., "Investigations on the Flow around Wheels using Different Road Simulation Tools", Progress in Vehicle Aerodynamics and Thermal Management, (Stuttgart, Germany, 2013): 155-166, ISBN: 978-3-8169-3253-6.

[12] Wittemeier, F, Willey, P, Kuthada, T, Widdecke, N, and Wiedemann, J, "Classification of Aerodynamic Tyre Characteristics", International Vehicle Aerodynamics Conference 2014, (Holywell Park, Loughborough, UK, 2014): 175-185, ISBN: 978-0-08-100199-8

[13] Schnepf, B., Schütz, T., and Indinger, T., "Further Investigations on the Flow Around a Rotating, Isolated Wheel with Detailed Tread Pattern”, SAE Int. J. Passeng. Cars - Mech. Syst. 8: 261-274, Apr. 2015, DOI: 10.4271/2015-01-1554.

[14] Lew, C., Gopalaswamy, N., Shock, R., Duncan, B., and Hoch, J., “Aerodynamic Simulation of a Standalone Rotating Treaded Tire”, WCX17: SAE World Congress Experience, (SAE International, Apr. 2017), DOI: 10.4271/2017-01-1551.

[15] Hobeika, T., Sebben, S., and Lofdahl, L., "Study of Different Tyre Simulation Methods and Effects on Passenger Car Aerodynamics", International Vehicle Aerodynamics Conference 2014, (Holywell Park, Loughborough, UK, 2014): 187-195, ISBN: 978-0-08-100199-8.

[16] Gullberg, P. V., "Optimisation of the Flow Process in Engine Bays-3D Modelling of Cooling Airflow”, PhD thesis, Gothenburg, Sweden, 2011: 239-245, ISBN: 978-91-7385-559-4.

[17] Lee, M.-H., Shiah, Y., and Bai, C.-J., "Experiments and numerical simulations of the rotor-blade performance for a small-scale horizontal axis wind turbine", Journal of Wind Engineering and Industrial Aerodynamics 149: 17 -29, 2016, ISSN: 0167-6105, DOI: http://dx.doi.org/10.1016/j.jweia.2015.12.002.

[18] Shur, M. L., Spalart, P. R., Strelets, M. K., and Travin, A. K., "A hybrid RANS-LES approach with delayed-DES and wall-modelled LES capabilities", International Journal of Heat and Fluid Flow 29(6): 1638 -1649, 2008, ISSN: 0142-727X, DoI: https://doi.org/10.1016/j.ijheatfluidflow.2008.07.001.

[19] Sterneus, J., Walker, T., and Bender, T., "Upgrade of the Volvo Cars Aerodynamic Wind Tunnel”, SAE Technical Paper, (Detroit, Michigan, SAE International, 2007), DoI: $\underline{10.4271 / 2007-01-1043}$

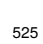

\section{Contact Information}

Dr. Simone Sebben

Road Vehicle Aerodynamics Group

Division of Vehicle Engineering and Autonomous Systems

Department of Mechanics and Maritime Sciences

Chalmers University of Technology, Sweden

Tel. $+46(0)$

simone.sebben@chalmers.se 
Mr. Teddy Hobeika

Road Vehicle Aerodynamics Group

\section{Division of Vehicle Engineering and Autonomous Systems}

Department of Mechanics and Maritime Sciences

Chalmers University of Technology, Sweden

Tel. $+46(0) 700738996$

teddy.hobeika@chalmers.se

\section{Acknowledgments}

The simulations were performed on resources provided by the Swedish

National Infrastructure for Computing (SNIC) at PDC Center for High

Performance Computing (PDC-HPC). The authors would also like to thank Volvo Cars for providing access to their test facility and resources.

\section{Funding}

This work is funded by Energimyndigheten (Swedish Energy Agency) project number 37195-1.

\section{Nomenclature}

\begin{tabular}{ll} 
Symbol & Definition \\
\hline \hline CFD & Computational Fluid Dynamics \\
RW & Rotation Wall \\
MRF & Moving Reference Frame \\
MRFg & Moving Reference Frame - grooves \\
SM & Sliding Mesh \\
IDDES & Improved Delayed Detached Eddy Simulation \\
C $_{d}$ & Drag Coefficient \\
S & Slick tyre \\
G & Lateral grooved tyre \\
D & Detailed tyre \\
CR & Closed Rim \\
OR & Open Rim \\
\hline
\end{tabular}

